\title{
The polynomial inverse image method
}

Vilmos Totik

\begin{abstract}
In this survey we discuss how to transfer results from an interval or the unit circle to more general sets. At the basis of the method is taking polynomial inverse images.
\end{abstract}

\section{Introduction}

In the last decade a method has been developed that (in some cases) allows one to transfer result from an interval (like $[-1,1]$ ) or the unit circle $C_{1}$ (which we are going to call model cases) to more general sets. We emphasize that the method TRANSFORMS the RESULT from the model case to the general case and is not aimed to carry over the proofs from the model cases to the general situation.

The rationale of the method is the following: on the unit circle $C_{1}$ and on $[-1,1]$ many classical and powerful tools (such as Fourier-series, classical orthogonal expansions, Poisson representation, Taylor expansions, $H^{p}$-spaces etc.) have been developed, which are at our disposal when dealing with a problem on these model sets. When dealing with more general sets like a compact subset of the real line instead of $[-1,1]$ or a system of Jordan corves instead of $C_{1}$, either these tools are nonexistent, or they are dif£cult to use. Therefore, if we have a method that transforms a model result to the general case, then

- we get the same result in many situations (as opposed to the single result in the model case),

- we are saved the burden of £nding the analogue of the model proof (which may not exist at all).

Vilmos Totik

Bolyai Institute, Analysis Research Group of the Hungarian Academy of Sciences, University of Szeged, Szeged, Aradi v. tere 1, 6720, Hungary, and Department of Mathematics and Statistics, University of South Florida 4202 E. Fowler Ave, PHY 114 Tampa, FL 33620-5700, USA, e-mail: totik@mail.usf.edu 
The method in question is the following: apply inverse images under polynomial mapping, i.e. if $T_{N}(z)=\gamma_{N} z^{N}+\cdots$ is a polynomial and $E_{0}$ is $[-1,1]$ or the unit circle $C_{1}$, then consider

$$
E=T_{N}^{-1} E_{0}=\left\{z \mid T_{N}(z) \in E_{0}\right\} .
$$

The point is that many properties are preserved when we take polynomial inverse images, most notable, equilibrium measures and Green's functions (see the Appendix) are preserved.

Thus, in a nutshell we make the following steps:

(a) Start from a result for the model case.

(b)Apply an inverse polynomial mapping to go to a special result on the inverse images of the model sets.

(c) Approximate more general sets by inverse images as in (b).

Sometimes, (b)-(c) should be followed by an additional step:

(d)Get rid of the special properties appearing in steps (b)-(c).

Among others the polynomial inverse image method has been successful in the following situations:

1. The Bernstein-type inequality (2) below, the model case being the classical Bernstein inequality (1) on $[-1,1]$.

2. The Markoff-type inequality (16)-(17) below, the model case being the classical Markoff inequality (15).

3. Asymptotics of Christoffel functions on compact subsets of the real line, namely (25), when the model case was $(23)$ on $[-1,1]$.

4. Asymptotics of Christoffel functions on curves, namely (26), when the model case was (22) on $C_{1}$.

5. Universality (28) on general sets, the model case being (28) on $[-1,1]$.

6. Fine zero spacing (30) of orthogonal polynomials, the model case being (29) on $[-1,1]$.

7. For a system of smooth Jordan curves the Bernstein-type inequality (19), where the model case was Bernstein's inequality (18) on the unit circle.

Before elaborating more on the method let us see how it works in a concrete case. To this we need a few things from potential theory; see the Appendix at the end of this paper for the de£nitions. In what follows, for a compact set $E \subset \mathbf{R}$ of positive capacity we denote by $\omega_{E}$ the density of the equilibrium measure with respect to the Lebesgue measure on $\mathbf{R}$. This density certain exists in the (one dimensional) interior of $E$. On the other hand, if $E$ is a £nite family of smooth Jordan curves or arcs, then $\omega_{E}$ denotes the density of the equilibrium measure of $E$ with respect to arc measure on $E$. 


\section{The Bernstein inequality on general sets}

Let $P_{n}$ denote an algebraic polynomial of degree at most $n$. Bernstein's inequality

$$
\left|P_{n}^{\prime}(x)\right| \leq \frac{n}{\sqrt{1-x^{2}}}\left\|P_{n}\right\|_{[-1,1]}, \quad x \in[-1,1]
$$

relating the derivative of $P_{n}$ to its supremum norm on $[-1,1]$ is of fundamental importance in approximation theory. Now with the polynomial inverse image method we can prove the following generalization of (1):

Theorem 2.1 If $E \subset \mathbf{R}$ is compact, then

$$
\left|P_{n}^{\prime}(x)\right| \leq n \pi \omega_{E}(x)\left\|P_{n}\right\|_{E}, \quad x \in \operatorname{Int}(E) .
$$

Note that for $E=[-1,1]$ we have $\omega_{E}(x)=1 / \pi \sqrt{1-x^{2}}$, so in this case (2) takes the form (1). Let us also mention that (2) is sharp: if $x_{0} \in \operatorname{Int}(E)$ is arbitrary, then for every $\varepsilon>0$ there are polynomials $P_{n}$ of degree at most $n=1,2, \ldots$ such that

$$
\left|P_{n}^{\prime}\left(x_{0}\right)\right|>(1-\varepsilon) n \pi \omega_{E}\left(x_{0}\right)\left\|P_{n}\right\|_{E}
$$

for all large $n$.

Actually, more is true, namely

$$
\left(\frac{\left|P_{n}^{\prime}(x)\right|}{\pi \omega_{E}(x)}\right)^{2}+n^{2}\left|P_{n}(x)\right|^{2} \leq n^{2}\left\|P_{n}\right\|_{E}^{2}, \quad x \in \operatorname{Int}(E),
$$

which is the analogue of the inequality

$$
\left(\left|P_{n}^{\prime}(x)\right| \sqrt{1-x^{2}}\right)^{2}+n^{2}\left|P_{n}(x)\right|^{2} \leq n^{2}\left\|P_{n}\right\|_{[-1,1]}^{2}
$$

of Szegb ([36],[6]).

(2) and (3) are due to M. Baran [1], who actually got them also in higher dimension. Both inequalities were rediscovered in [39] with the method of the present survey. The outline of the proof of (2) using polynomial inverse images is as follows:

(a) Start from Bernstein inequality on $[-1,1]$.

(b)Next consider the special case when $E=T_{N}^{-1}[-1,1]$ and $P_{n}=S_{k}\left(T_{N}\right)$ with some polynomial $S_{k}$. Assuming $\left\|P_{n}\right\|_{E}=1$ we get

$$
\left|P_{n}^{\prime}(x)\right|=\left|S_{k}^{\prime}\left(T_{N}(x)\right) T_{N}^{\prime}(x)\right| \leq \frac{k}{\sqrt{1-T_{N}^{2}(x)}}\left|T_{N}^{\prime}(x)\right|=k N \pi \frac{\left|T_{N}^{\prime}(x)\right|}{\pi N \sqrt{1-T_{N}^{2}(x)}},
$$

and by (6) here the right-hand side is $k N \pi \omega_{E}(x)$, i.e. we get (2) in this special case.

(c) Approximate a general $E$ by $T_{N}^{-1}[-1,1]$ and $P_{n}$ by $S_{k}\left(T_{N}\right)$ to get 


$$
\left|P_{n}^{\prime}(x)\right| \leq\left(1+o_{x, E}(1)\right) n \pi \omega_{E}(x)\left\|P_{n}\right\|_{E}
$$

where $o_{x, E}(1)$ denotes a quantity that tends to 0 as $n$ tends to infnity. See section 5 for this approximation step (the exact details for the general Bernstein inequality are in [39, Theorem 3.1]).

(d) Get rid of $o(1)$.

This very last step can be done as follows. Let $P_{n}$ be any polynomial, and $x_{0}$ any point in the interior of $E$. We may assume $\left\|P_{n}\right\|_{E}=1$. Let $\mathscr{T}_{m}(z)=\cos (m \arccos z)$ be the classical Chebyshev polynomials, and for some $0<\alpha_{m}<1$ and $0 \leq \varepsilon_{m}<1-\alpha_{m}$ consider the polynomials

$$
R_{m n}(x)=\mathscr{T}_{m}\left(\alpha_{m} P_{n}(x)+\varepsilon_{m}\right)
$$

where $\alpha_{m}<1$ and $0 \leq \varepsilon_{m}<1-\alpha_{m}$ are chosen so that $\alpha_{m} P_{n}\left(x_{0}\right)+\varepsilon_{m}$ is one of the zeros of $\mathscr{T}_{m}$. Since the distance of neighboring zeros of $\mathscr{T}_{m}$ is smaller than $10 / m$, we can do this with $\alpha_{m}=1-10 / m$ and with some $0 \leq \varepsilon_{m}<10 / m$, and then $\alpha_{m} \rightarrow 1$ and $\varepsilon_{m} \rightarrow 0$ as $m \rightarrow \infty$. Now apply (5) to $R_{m n}$. It follows that

$$
\left|R_{m n}^{\prime}\left(x_{0}\right)\right| \leq(1+o(1)) \pi \omega_{E}\left(x_{0}\right) m n\left\|R_{m n}\right\|_{E},
$$

where the term $o(1)$ tends to zero as $m \rightarrow \infty$. Here, on the right, $\left\|R_{m n}\right\|_{E}=1$, and on the left we have

$$
\left|R_{m n}^{\prime}\left(x_{0}\right)\right|=\left|\mathscr{T}_{m}^{\prime}\left(\alpha_{m} P_{n}\left(x_{0}\right)+\varepsilon_{m}\right)\right|\left|P_{n}^{\prime}\left(x_{0}\right)\right| \alpha_{m}
$$

Since at the zeros $z$ of $\mathscr{T}_{m}$ we have $\mathscr{T}_{m}^{\prime}(z)=m / \sqrt{1-z^{2}}$, it follows that

$$
\frac{m}{\sqrt{1-\left(\alpha_{m} P_{n}\left(x_{0}\right)+\varepsilon_{m}\right)^{2}}}\left|P_{n}^{\prime}\left(x_{0}\right)\right| \alpha_{m} \leq(1+o(1)) \pi \omega_{E}\left(x_{0}\right) m n,
$$

where the term $o(1)$ tends to zero as $m \rightarrow \infty$. On dividing here by $m$ and letting $m$ tend to infnity we obtain

$$
\frac{\left|P_{n}^{\prime}\left(x_{0}\right)\right|}{\sqrt{1-P_{n}^{2}\left(x_{0}\right)}} \leq \pi \omega_{E}\left(x_{0}\right) n,
$$

and this is the inequality (3) at the point $x_{0}$ because in our case $\left\|P_{n}\right\|_{E}=1$.

\section{The model case $[-1,1]$, admissible polynomial maps, approximation}

As we have already mentioned, there are two model cases: the interval $[-1,1]$ and the unit circle $C_{1}=\{z|| z \mid=1\}$. 
For $[-1,1]$ we allow polynomial maps with respect to real polynomials (called admissible polynomials) $T_{N}(z)=\gamma_{n} x^{N}+\cdots, \gamma_{N} \neq 0$ such that $T_{N}$ has $N$ zeros and $N-1$ local extremal values each of which is of size $\geq 1$ in absolute value. In other words, there are $u_{1}, \ldots, u_{N}$ with $T_{N}^{\prime}\left(u_{j}\right)=0$ and $\left|T_{N}\left(u_{j}\right)\right| \geq 1$. Then it easily follows that the local extremal values alternate in sign and $T_{N}(z)$ runs through the interval $[-1,1] N$-times as $x$ runs through the real line. Thus,

$$
E:=T_{N}^{-1}[-1,1]=\left\{x \mid T_{N}(x) \in[-1,1]\right\}
$$

consists of $N$ subintervals $E_{n, j}, 1 \leq j \leq N$ each of which is mapped by $T_{N}$ onto $[-1,1]$ in a 1-to- 1 fashion. However, some of these subintervals may be attached to one another, so $T_{N}^{-1}[-1,1]$ actually consists of $k$ intervals for some $1 \leq k \leq N$; see Figure 1 where $N=6$ and $k=3$. The equilibrium measure of $E$ is the (normalized) pull-back of the equilibrium measure on $[-1,1]$ under the mapping $T_{N}$ :

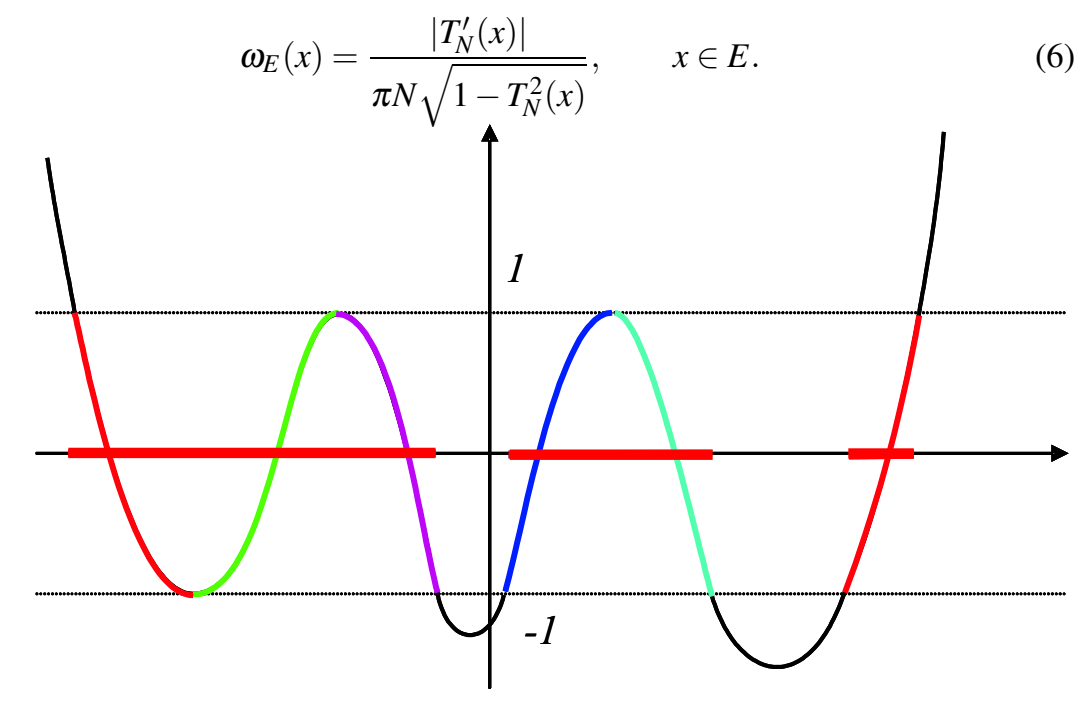

Fig. 1

Polynomial inverse images of intervals, i.e. sets of the form $T_{N}^{-1}[-1,1]$ with admissible $T_{N}$ have many interesting properties. They are the sets $\Sigma=\cup_{j=1}^{l}\left[a_{j}, b_{j}\right]$ with the property that the equilibrium measure has rational mass on each subinterval, i.e. each $\mu_{\Sigma}\left(\left[a_{j}, b_{j}\right]\right), j=1, \ldots, k$ is of the form $p / N$. They are also the sets $\Sigma=\cup_{i=1}^{l}\left[a_{i}, b_{i}\right]$ for which the Pell-type equation

$$
P^{2}(z)-Q(z) S^{2}(z)=1 \quad \text { with } \quad Q(x)=\prod_{i=1}^{l}\left(x-a_{i}\right)\left(x-b_{i}\right),
$$


which goes back to N. H. Abel, has polynomial solutions $P$ and $Q$. See [24] - [29] and the references there for many more interesting results connected with polynomial inverse images.

What we need of them is that these sets are dense among all sets consisting of £nitely many intervals.

Theorem 3.1 Given a system $\Sigma=\left\{\left[a_{i}, b_{i}\right]\right\}_{i=1}^{l}$ of disjoint closed intervals and an $\varepsilon>0$, there is another system $E=\left\{\left[a_{i}^{\prime}, b_{i}^{\prime}\right]\right\}_{i=1}^{l}$ such that $\cup_{i=1}^{l}\left[a_{i}^{\prime}, b_{i}^{\prime}\right]=T_{N}^{-1}[-1,1]$ for some admissible polynomial $T_{N}$, and for each $1 \leq i \leq l$ we have

$$
\left|a_{i}-a_{i}^{\prime}\right| \leq \varepsilon, \quad\left|b_{i}-b_{i}^{\prime}\right| \leq \varepsilon .
$$

The theorem immediately implies its strengthened form when we also prescribe if a given $a_{i}^{\prime}$ (or $b_{i}^{\prime}$ ) is smaller or bigger than $a_{i}$ (or $b_{i}$ ). In particular, it is possible to require e.g. that $\Sigma \subset \Sigma^{\prime}$. It is also true that in the theorem we can select $a_{i}^{\prime}=a_{i}$ for all $i$, and even $b_{l}^{\prime}=b_{l}$. Alternatively we can $£ x$ any $l+1$ of the $2 l$ points $a_{i}, b_{i}$, $1 \leq i \leq l$.

Theorem 3.1 has been proven several times independently in the literature, see [31], [19], [7], [39], [23]. For a particularly simple proof see [42].

\section{The model case $C_{1}$, sharpened form of Hilbert's lemniscate theorem}

For the unit circle $C_{1}$ we shall take its inverse image under polynomial mappings generated by polynomials $T_{N}(z)=\gamma_{N} z^{N}+\cdots$ for which $T_{N}^{\prime}(z) \neq 0$ whenever $\left|T_{N}(z)\right|=1$. Then

$$
\sigma:=T_{N}^{-1} C_{1}=\left\{z|| T_{N}(z) \mid=1\right\}
$$

is actually a level set of the polynomial $T_{N}$, which, from now on, we call a lemniscate. Since $T_{N}^{\prime}(z) \neq 0$ on $E$, this $E$ consists of a £nite number of analytic Jordan curves (a Jordan curve is a homeomorphic image of the unit circle). Again, the equilibrium measure of $E$ is the (normalized) pull-back of the equilibrium measure on $C_{1}$ under the mapping $T_{N}$ :

$$
\omega_{\sigma}(z)=\frac{1}{2 \pi N}\left|T_{N}^{\prime}(z)\right|, \quad z \in E
$$

Hilbert's lemniscate theorem claims that if $K$ is a compact set on the plane and $U$ is a neighborhood of $K$ then there is a lemniscate $\sigma$ that separates $K$ and $\mathbf{C} \backslash U$, i.e. it lies within $U$ but encloses $K$. An equivalent formulation is the following. Let $\gamma_{j}, \Gamma_{j}, j=1, \ldots, m$ be Jordan curves (i.e. homeomorphic images of the unit circle), $\gamma_{j}$ lying interior to $\Gamma_{j}$ and the $\Gamma_{j}$ 's lying exterior to one another, and set $\gamma^{*}=\cup_{j} \gamma_{j}$, $\Gamma^{*}=\cup_{j} \Gamma_{j}$. Then there is a lemniscate $\sigma$ that is contained in the interior of $\Gamma^{*}$ which also contains $\gamma^{*}$ in its interior, i.e. $\sigma$ separates $\gamma^{*}$ and $\Gamma^{*}$ in the sense that it 
separates each $\gamma_{j}$ from the corresponding $\Gamma_{j}$. This is not enough for our purposes of approximation, what we need is the following sharpened form (see [20]).

Let $\gamma^{*}$ and $\Gamma^{*}$ be twice continuously differentiable in a neighborhood of $P$ and touching each other at $P$. We say that they $\mathscr{K}$-touch each other if their (signed) curvature at $P$ is different (signed curvature is seen from the outside of $\Gamma^{*}$ ). Equivalently we can say that in a neighborhood of $P$ the two curves are separated by two circles one of them lying in the interior of the other one.

Theorem 4.1 Let $\gamma^{*}=\cup_{j=1}^{m} \gamma_{j}$ and $\Gamma^{*}=\cup_{j=1}^{m} \Gamma_{j}$ be as above, and let $\gamma^{*} \mathscr{K}$-touch $\Gamma^{*}$ in Enitely many points $P_{1}, \ldots, P_{k}$ in a neighborhood of which both curves are twice continuously differentiable. Then there is a lemniscate $\sigma$ that separates $\gamma^{*}$ and $\Gamma^{*}$ and $\mathscr{K}$-touches both $\gamma^{*}$ and $\Gamma^{*}$ at each $P_{j}$.

Furthermore, $\sigma$ lies strictly in between $\gamma^{*}$ and $\Gamma^{*}$ except for the points $P_{1}, \ldots, P_{k}$, and has precisely one connected component in between each $\gamma_{j}$ and $\Gamma_{j}, j=1, \ldots, m$, and these $m$ components are Jordan curves.

From our point of view the following corollary is of primary importance. Let $K$ be the closed set enclosed by $\Gamma^{*}$ and $K_{0}$ the closed set enclosed by $\gamma^{*}$. Denote by $g(K, z)$ Green's function of $\overline{\mathbf{C}} \backslash K$ with pole at infnity. Finally, let $L$ be the closed set enclosed by $\sigma$.

Corollary 4.2 Let $\Gamma^{*}, \gamma^{*}$ and $P_{1}, \ldots, P_{k} \in \Gamma^{*}$ be as in Theorem 4.1. Then for every $\varepsilon>0$ there is a lemniscate $\sigma$ as in Theorem 4.1 such that for each $P_{j}$ we have

$$
\frac{\partial g\left(L, P_{j}\right)}{\partial \mathbf{n}} \leq \frac{\partial g\left(K, P_{j}\right)}{\partial \mathbf{n}}+\varepsilon
$$

where $\partial(\cdot) / \partial \mathbf{n}$ denotes (outward) normal derivative.

In a similar manner, for every $\varepsilon>0$ there is a lemniscate $\sigma$ as in Theorem 4.1 such that for each $P_{j}$ we have

$$
\frac{\partial g\left(K_{0}, P_{j}\right)}{\partial \mathbf{n}} \leq \frac{\partial g\left(L, P_{j}\right)}{\partial \mathbf{n}}+\varepsilon
$$

Note that

$$
\frac{\partial g\left(K, P_{j}\right)}{\partial \mathbf{n}} \leq \frac{\partial g\left(L, P_{j}\right)}{\partial \mathbf{n}} \leq \frac{\partial g\left(K_{0}, P_{j}\right)}{\partial \mathbf{n}},
$$

because $K_{0} \subset L \subset K$.

Now $\partial g\left(K, P_{j}\right) / \partial \mathbf{n}$ gives $2 \pi$-times the density of the equilibrium measure at $P_{j}$ with respect to arc length on $\Gamma^{*}$ :

$$
\omega_{\Gamma^{*}}\left(P_{j}\right)=\frac{1}{2 \pi} \frac{\partial g\left(K, P_{j}\right)}{\partial \mathbf{n}},
$$

hence we can reformulate (with a different $\varepsilon$ ) (8) as

$$
\omega_{\sigma}\left(P_{j}\right) \leq \omega_{\Gamma^{*}}\left(P_{j}\right)+\varepsilon
$$

and similarly, (9) can be reformulated as 


$$
\omega_{\gamma^{*}}\left(P_{j}\right) \leq \omega_{\sigma}\left(P_{j}\right)+\varepsilon
$$

\section{A critical point in the method}

The splitting of the set appears in the step (b) when we go from the model case to its inverse image under a polynomial mapping. That is a big advance, since from then on one works with several components, and they may be suffciently general to imitate an arbitrary set. However, there is a huge price to pay, namely in the transfer, say, from $[-1,1]$ to $E=T_{N}^{-1}[-1,1]$, the result is transferred into a very special statement on $E$, e.g. in the Bernstein inequality (1) in this step we got the extension (2) of the Bernstein inequality on $E$, but only for very special polynomials, namely of the form $Q_{k}\left(T_{N}\right)$. But our aim is to prove (in this case) the full analogue for ALL polynomials. Besides, in $Q_{k}\left(T_{N}\right)$ the polynomial $T_{N}$ is not known, and when we approximate an arbitrary set of £nitely many intervals by $T_{N}^{-1}[-1,1]$, it is typically of very high degree.

The idea of how to get rid of the special properties is the following. As we have already observed, $T_{N}^{-1}[-1,1]$ consists of $N$ subintervals $E_{i}=E_{N, i}$, and we denote by $T_{N, i}^{-1}$ that branch of $T_{N}^{-1}$ that maps $[-1,1]$ into $E_{i}$. Let $P_{n}$ be an arbitrary polynomial of degree $n$, and consider the sum

$$
S(x)=\sum_{i=1}^{N} P_{n}\left(T_{N, i}^{-1}\left(T_{N}(x)\right)\right) .
$$

We claim that this is a polynomial of $T_{N}(x)$ of degree at most $n / N$, i.e. $S(x)=$ $S_{n}\left(T_{N}(x)\right)$ for some polynomial $S_{n}$ of degree at most $n / N$. To this end let $x_{i}=$ $T_{N, i}^{-1}\left(T_{N}(x)\right), i=1, \ldots, N$. Then

$$
S(x)=S\left(x_{1}, \ldots, x_{N}\right)=\sum_{i=1}^{N} P_{n}\left(x_{i}\right)
$$

is a symmetric polynomial of the variables $x_{1}, \ldots, x_{N}$, and hence it is a polynomial of the elementary symmetric polynomials

$$
S_{j}\left(x_{1}, \ldots, x_{N}\right)=\sum_{1 \leq k_{1}<k_{2}<\ldots<k_{j} \leq N} x_{k_{1}} x_{k_{2}} \cdots x_{k_{j}}, \quad 1 \leq j \leq N .
$$

However, $x_{1}, x_{2}, \ldots, x_{N}$ are the roots in $t$ of the polynomial equation $T_{N}(t)=T_{N}(x)$, and so if $T_{N}(x)=d_{N} x^{N}+\cdots+d_{0}$, then it follows that

$$
S_{j}\left(x_{1}, \ldots, x_{N}\right)=(-1)^{j} d_{N-j} / d_{N}
$$

if $1 \leq j<N$, while

$$
S_{N}\left(x_{1}, \ldots, x_{N}\right)=(-1)^{N}\left(d_{0}-T_{N}(x)\right) / d_{N},
$$


from which the claim that $S$ is a polynomial of $T_{N}(x)$ follows. On comparing the degree of the homogeneous parts of these polynomials, we can see that the degree of

$$
S_{n}(u):=S\left(T_{N, 1}^{-1}(u)\right)
$$

is at $\operatorname{most} \operatorname{deg}\left(P_{n}\right) / N \leq n / N$ in $u$.

There is a slight problem, namely if $x \in E_{N, i_{0}}$, then the sum $S(x)$ contains not only $P_{n}(x)$, but also the values of $P_{n}$ at the conjugate points $x_{i}=T_{N, i}^{-1}\left(T_{N}(x)\right)$, so $S(x)$ does not really behave like $P_{n}(x)$. But that is easy to correct, namely we do not form $S$ from $P_{n}$, but rather from a $P_{n}^{*}$, which behaves like $P_{n}$ around $x$ and is small at conjugate points. To illustrate this crucial step, we complete the proof of (5) in the transform of the Bernstein inequality.

Let $\varepsilon>0$ be arbitrary. Then, by Theorem 3.1, there are polynomial inverse image sets $E^{*}$ consisting of the same number of intervals as $E$ such that the corresponding endpoints of the subintervals of $E$ and $E^{*}$ are as close as we wish. Therefore, we can choose $E^{*} \subset \operatorname{Int}(E)$ so that

$$
\omega_{E^{*}}\left(x_{0}\right) \leq(1+\varepsilon) \omega_{E}\left(x_{0}\right)
$$

is satisfed. Let $E^{*}=T_{N}^{-1}[-1,1]$, and let $E_{i}^{*}=T_{N, i}^{-1}[-1,1], i=1, \ldots, N$ be the $N$ inverse image intervals of $[-1,1]$ under the $N$ branches of $T_{N}^{-1}$. Since any translate of $E^{*}$ is the polynomial inverse image of $[-1,1]$ via a translate of $T_{N}$, we can assume without loss of generality that $x_{0}$ is not an endpoint of any of the intervals $E_{i}^{*}$, i.e. $x_{0}$ is lying in the interior of $E_{i_{0}}^{*}$ for some $i_{0}$.

Let $P_{n}$ be an arbitrary polynomial of degree $n$, and consider the polynomial

$$
P_{n}^{*}(x)=\left(1-\alpha\left(x-x_{0}\right)^{2}\right)^{[\sqrt{n}]} P_{n}(x),
$$

where $\alpha>0$ is £xed so that $1-\alpha\left(x-x_{0}\right)^{2}>0$ on $E$. Clearly, $P_{n}^{*}$ has degree at $\operatorname{most} n+2 \sqrt{n},\left\|P_{n}^{*}\right\|_{E} \leq\left\|P_{n}\right\|_{E}, P_{n}^{*}\left(x_{0}\right)=P_{n}\left(x_{0}\right),\left(P_{n}^{*}\right)^{\prime}\left(x_{0}\right)=P_{n}^{\prime}\left(x_{0}\right)$, and there is a $0<\beta<1$ such that

$$
\left|P_{n}^{*}(x)\right| \leq \beta^{\sqrt{n}}\left\|P_{n}\right\|_{E}, \quad\left|\left(P_{n}^{*}(x)\right)^{\prime}\right| \leq \beta^{\sqrt{n}}\left\|P_{n}\right\|_{E}
$$

uniformly for $x \in E \backslash E_{i_{0}}^{*}$ (for the last relations just observe that the factor $1-\alpha(x-$ $\left.x_{0}\right)^{2}$ is nonnegative and strictly less than one on $\left.E \backslash E_{i_{0}}^{*}\right)$. For $x \in E^{*}$ form now

$$
S(x)=\sum_{i=1}^{N} P_{n}^{*}\left(T_{N, i}^{-1}\left(T_{N}(x)\right)\right) .
$$

As we have already observed, this is a polynomial of degree at most $(n+2 \sqrt{n}) / N$ of $T_{N}(x)$, i.e. $S(x)=S_{n}\left(T_{N}(x)\right)$ for some polynomial $S_{n}$ of degree at most $(n+2 \sqrt{n}) / N$. From the properties (13) it is also clear that

$$
\|S\|_{E^{*}} \leq\left(1+N \beta^{\sqrt{n}}\right)\left\|P_{n}\right\|_{E}, \quad\left|S^{\prime}\left(x_{0}\right)-P_{n}^{\prime}\left(x_{0}\right)\right| \leq N \beta^{\sqrt{n}}\left\|P_{n}\right\|_{E}
$$


Now $S$ is already of the type for which we have veri£ed (2) above, so if we apply to $S$ the inequality (2) at $x=x_{0}$, and if we use (11) and the preceding estimates we obtain (2):

$$
\begin{aligned}
\left|P_{n}^{\prime}\left(x_{0}\right)\right| & \leq\left|S^{\prime}\left(x_{0}\right)\right|+N \beta^{\sqrt{n}}\left\|P_{n}\right\|_{E} \\
& \leq(n+2 \sqrt{n}) \pi \omega_{E^{*}}\left(x_{0}\right)\|S\|_{E^{*}}+N \beta^{\sqrt{n}}\left\|P_{n}\right\|_{E} \\
& \leq(n+2 \sqrt{n})(1+\varepsilon) \pi \omega_{E}\left(x_{0}\right)\left(1+N \beta^{\sqrt{n}}\right)\left\|P_{n}\right\|_{E}+N \beta^{\sqrt{n}}\left\|P_{n}\right\|_{E} \\
& =(1+o(1)) n \pi \omega_{E}\left(x_{0}\right)\left\|P_{n}\right\|_{E},
\end{aligned}
$$

since $\varepsilon>0$ was arbitrary.

\section{The Markoff inequality for several intervals}

The classical Markoff inequality

$$
\left\|P_{n}^{\prime}\right\|_{[-1,1]} \leq n^{2}\left\|P_{n}\right\|_{[-1,1]}
$$

complements Bernstein's inequality when we have to estimate the derivative of a polynomial on $[-1,1]$ close to the endpoints. What happens, if we consider more than one intervals? In [8] it was shown that if $E=[-b,-a] \cup[a, b]$, then

$$
\left\|P_{n}^{\prime}\right\|_{E} \leq(1+o(1)) \frac{n^{2} b}{b^{2}-a^{2}}\left\|P_{n}\right\|_{E} .
$$

Why is $b /\left(b^{2}-a^{2}\right)$ the correct factor here? This can be answered by the transformation $x \rightarrow x^{2}$, but what if we have two intervals of different size, or when we have more than two intervals? With the polynomial inverse image method we proved in [39] the following extension.

Let $E=\cup_{j=1}^{l}\left[a_{2 j-1}, a_{2 j}\right], a_{1}<a_{2}<\cdots<a_{2 l}$ consist of $l$ intervals. When we consider the analogue of the Markoff inequality for $E$, actually we have to talk about one-one Markoff inequality around every endpoint of $E$. Let $a_{j}$ be an endpoint of $E, E^{j}$ part of $E$ that lies closer to $a_{j}$ than to any other endpoint. Let $M_{j}$ be the best constant for which

$$
\left\|P_{n}^{\prime}\right\|_{E^{j}} \leq(1+o(1)) M_{j} n^{2}\left\|P_{n}\right\|_{E}
$$

holds, where $o(1)$ tends to 0 as $n$ tends to in£nity. This $M_{j}$ clearly depends on what endpoint $a_{j}$ we are considering. Its value is given by (see [39])

\section{Theorem 6.1}

$$
M_{j}=2 \frac{\prod_{i=1}^{l-1}\left(a_{j}-\lambda_{i}\right)^{2}}{\prod_{i \neq j}\left|a_{j}-a_{i}\right|},
$$

where the $\lambda_{j}$ are the numbers that appear in the equilibrium measure in (40)-(41). 
Let us consider the example $E=[-b,-a] \cup[a, b]$. In this case $l=2, a_{1}=$ $-b, a_{2}=-a, a_{3}=a, a_{4}=b$, and, by symmetry, $\lambda_{1}=0$. Hence

$$
\begin{gathered}
\omega_{E}(t)=\frac{|t|}{\pi \sqrt{\left(b^{2}-t^{2}\right)\left(t^{2}-a^{2}\right)}}, \\
M_{1}=M_{4}=\frac{2 b^{2}}{(b-a)(b+a)(2 b)}=\frac{b}{b^{2}-a^{2}} \\
M_{2}=M_{3}=\frac{2 a^{2}}{(b-a)(b+a)(2 b)}=\frac{a}{b^{2}-a^{2}} .
\end{gathered}
$$

Since $M_{1}=M_{4}>M_{2}=M_{3}$ we obtain that

$$
\left\|P_{n}^{\prime}\right\|_{[-b,-a] \cup[a, b]} \leq(1+o(1)) n^{2} \frac{b}{b^{2}-a^{2}}\left\|P_{n}\right\|_{[-b,-a] \cup[a, b]},
$$

which is the result of [8] mentioned above.

As an immediate consequence of the theorem we get the following asymptotically best possible Markoff inequality:

\section{Corollary 6.2}

$$
\left\|P_{n}^{\prime}\right\|_{E} \leq(1+o(1)) n^{2}\left(\max _{1 \leq j \leq 2 l} M_{j}\right)\left\|P_{n}\right\|_{E}
$$

It is quite interesting that here the $o(1)$ term cannot be dropped. This is due to the strange fact that there are cases, where the maximum of

$$
\left|P_{n}^{\prime}(x)\right| /\left\|P_{n}\right\|_{E}
$$

for all $x \in E$ and all $P_{n}$ of given degree $n$, is attained in an inner point of $E$ ([2]).

It seems to be a diffcult problem to $£$ nd on several intervals for each $n$ the best Markoff constant for polynomials of degree at most $n$. The previous corollary gives the asymptotically best constant (as $n$ tends to infnity).

\section{Bernstein's inequality on curves}

Bernstein had another inequality on the derivative of a polynomial, namely if $C_{1}$ is the unit circle, then

$$
\left|P_{n}^{\prime}(z)\right| \leq n\left\|P_{n}\right\|_{C_{1}}, \quad z \in C_{1}
$$

for any polynomial of degree at most $n$. With the polynomial inverse image method in [20] we extended this to a family of $C^{2}$ Jordan curves.

Theorem 7.1 Let $E$ be a £nite union of $C^{2}$ Jordan curves (lying exterior to one another), and $\omega_{E}$ the density of the equilibrium measure of $E$ with respect to arc 
length. Then for any polynomial $P_{n}$ of degree at most $n=1,2, \ldots$

$$
\left|P_{n}^{\prime}(z)\right| \leq(1+o(1)) 2 \pi n \omega_{E}(z)\left\|P_{n}\right\|_{E}, \quad z \in E .
$$

This is sharp:

Theorem 7.2 With the assumptions of the previous theorem for any $z_{0} \in E$ there are polynomials $P_{n}$ of degree at most $n$ such that

$$
\left|P_{n}^{\prime}\left(z_{0}\right)\right|>(1-o(1)) 2 \pi n \omega_{E}\left(z_{0}\right)\left\|P_{n}\right\|_{E}
$$

for some $P_{n}$ 's.

We mention that the term $o(1)$ is necessary, without it the inequality is not true. Note also that, as opposed to (2), here, on the right hand side, the factor is $2 \pi \omega_{E}(z)$ rather than $\pi \omega_{E}(z)$.

Corollary 7.3 If $E$ is a £nite family of disjoint $C^{2}$ Jordan curves then

$$
\left\|P_{n}^{\prime}\right\|_{E} \leq(1+o(1)) n\left(2 \pi \sup _{z \in E} \omega_{E}(z)\right)\left\|P_{n}\right\|_{E},
$$

and this is sharp, for

$$
\left\|P_{n}^{\prime}\right\|_{E}>(1-o(1)) n\left(2 \pi \sup _{z \in E} \omega_{E}(z)\right)\left\|P_{n}\right\|_{E}
$$

for some polynomials $P_{n}, n=1,2, \ldots$

\section{Asymptotics for Christoffel functions}

Let $\mu$ be a £nite Borel measure on the plane such that its support is compact and consists of infnitely many points. The Christoffel functions associated with $\mu$ are de£ned as

$$
\lambda_{n}(\mu, z)=\inf _{P_{n}(z)=1} \int\left|P_{n}\right|^{2} d \mu
$$

where the infmum is taken for all polynomials of degree at most $n$ that take the value 1 at $z$. If $p_{k}(z)=p_{k}(\mu, z)$ denote the orthonormal polynomials with respect to $\mu$, i.e.

$$
\int p_{n} \overline{p_{m}} d \mu=\delta_{n, m}
$$

then $\lambda_{n}$ can be expressed as

$$
\lambda_{n}^{-1}(\mu, z)=\sum_{k=0}^{n}\left|p_{k}(z)\right|^{2}
$$


In other words, $\lambda_{n}^{-1}(z)$ is the diagonal of the reproducing kernel

$$
K_{n}(z, w)=\sum_{k=0}^{n} p_{k}(z) \overline{p_{k}(w)}
$$

which makes it an essential tool in many problems.

In past literature a lot of work has been devoted to Christoffel functions, e.g. the $H^{p}$ theory emerged from Szegø's theorem; the density of states in statistical mechanical models of quantum physics is given by the reciprocal of the Christoffel function associated with the spectral measure (see e.g. [22]); and the recent breakthrough [16] by Lubinsky in universality connected with random matrices has also been based on them (cf. also [10], [41] and particularly [32] where the importance of Christoffel functions regarding off diagonal behavior of the reproducing kernel was emphasized). See [12], [14], [34], and particularly [21] by P. Nevai and [33] by B. Simon for the role and various use of Christoffel functions.

In 1915 Szegd proved that if $d \mu(t)=\mu^{\prime}(t) d t$ is an absolutely continuous measure on the unit circle (identifed with $[-\pi, \pi]$ ) then

$$
\lim _{n \rightarrow \infty} \lambda_{n}(z)=\left(1-|z|^{2}\right) \exp \left(\frac{1}{2 \pi} \int_{-\pi}^{\pi} \frac{e^{i t}-z}{e^{i t}+z} \log \mu^{\prime}(t) d t\right), \quad|z|<1
$$

provided $\log \mu^{\prime}(t)$ is integrable (otherwise the limit on the left is 0 ). Just to show the importance of Christoffel functions, let us mention that the $z=0$ case of this theorem immediately implies that the polynomials are dense in $L^{2}(\mu)$ if and only if $\int \log \mu^{\prime}=-\infty$. Szegd ([37, Th. I', p. 461]) also proved that on the unit circle

$$
\lim _{n \rightarrow \infty} n \lambda_{n}\left(\mu, e^{i \theta}\right)=2 \pi \mu^{\prime}(\theta)
$$

under the condition that $\mu$ is absolutely continuous and $\mu^{\prime}>0$ is twice continuously differentiable. The almost everywhere result came much later, only in 1991 was it proven in [18] that (22) is true almost everywhere provided $\log \mu^{\prime}$ is integrable.

All the aforestated results can be translated into theorems on $[-1,1]$, e.g.: if the support of $\mu$ is $[-1,1]$ and $\log \mu^{\prime} \in L_{\mathrm{loc}}^{1}$, then

$$
\lim _{n \rightarrow \infty} n \lambda_{n}(x)=\pi \sqrt{1-x^{2}} \mu^{\prime}(x)
$$

almost everywhere. A local result is that (23) is true on an interval $I$ if $\mu$ is in the $\mathbf{R e g}$ class (see below), $\mu$ is absolutely continuous on $I$ and $\log \mu^{\prime} \in L^{1}(I)$. The measure $\mu$ is called to be in the Reg class (see [35, Theorem 3.2.3]) if the $L^{2}(\mu)$ and $L^{\infty}(\mu)$ norms of polynomials are asymptotically the same in $n$-th root sense:

$$
\limsup _{n \rightarrow \infty} \frac{\left\|Q_{n}\right\|_{L^{\infty}(\mu)}^{1 / n}}{\left\|Q_{n}\right\|_{L^{2}(\mu)}^{1 / n}} \leq 1
$$


An equivalent formulation is: $\lambda_{n}(\mu, z)^{1 / n} \rightarrow 1$ uniformly on the support of $\mu$. $\mu \in$ Reg is a fairly weak condition on $\mu$; see [35] for general regularity criteria and different equivalent formulations of $\mu \in \mathbf{R e g}$. For example, $\mu^{\prime}>0$ a.e. implies that $\mu \in$ Reg.

When the support is not $[-1,1]$, things change. Indeed, let $K=\operatorname{supp}(\mu) \subset \mathbf{R}$ be a compact set (of positive logarithmic capacity), and let $v_{K}$ denote the equilibrium measure of $K$. The polynomial inverse image method gives (see [38], [41])

Theorem 8.1 Let $K=\operatorname{supp}(\mu)$ be a compact set of positive capacity and suppose that $\mu \in \mathbf{R e g}$ and $\log \mu^{\prime} \in L^{1}(I)$ for some interval $I \subset K$. Then almost everywhere on $I$

$$
\lim _{n \rightarrow \infty} n \lambda_{n}(\mu, x)=\frac{d \mu(x)}{d v_{K}},
$$

where, on the right-hand side, the expression is the Radon-Nikodym derivative of $\mu$ with respect to the equilibrium measure $\mu_{K}$.

Of course, when $K=[-1,1]$, then (23) and (25) are the same.

In a similar vein, but with totally different proof (based now on the model case $C_{1}$ ) we have (see [43]):

Theorem 8.2 Let $K=\operatorname{supp}(\mu)$ be a £nite family of $C^{2}$ Jordan curves and suppose that $\mu \in \mathbf{R e g}$ and $\log \mu^{\prime} \in L^{1}(I)$ for some arc $I \subset K$. Then almost everywhere on $I$

$$
\lim _{n \rightarrow \infty} n \lambda_{n}(\mu, x)=\frac{d \mu(x)}{d v_{K}},
$$

Here $L^{1}(I)$ is meant with respect to arc measure on $K$.

We note that (26) holds at every point where the measure $\mu$ has continuous density with respect to arc length (see [43]). In this case the support of $\mu$ can be much more general, and the result is about the asymptotics of the Christoffel function on an outer boundary arc of the support.

One can also allow a combination of Jordan arcs (homeomorphic images of $[-1,1])$ and curves for the support of $\mu$. However, this extension does not come directly from the polynomial inverse image method, for there is a huge difference between smooth Jordan arcs and Jordan curves: the interior of Jordan curves (or family of curves) can be exhausted by lemniscates, and once an arc is in the set, this is no longer true.

Orthogonal polynomials with respect to area measures go back to Carleman [9] who gave strong asymptotics for them in the case of a Jordan domain with analytic boundary curve. For less smooth domains or for regions consisting of several components the situation is more dif£cult. The polynomial inverse image method in [43] gave the asymptotics for Christoffel functions with respect to area-like measures:

Theorem 8.3 Suppose that $K$ is a compact set bounded by a £nite number of $C^{2}$ Jordan curves and $\mu$ is a measure on $K$ of the form $d \mu=W d A$ with some continuous $W$ such that that

$$
\operatorname{cap}(\{z \mid W(z)>0\} \cap \operatorname{Int}(K))=\operatorname{cap}(K) .
$$


Then for $z_{0} \in \partial K$

$$
\lim _{n \rightarrow \infty} n^{2} \lambda_{n}\left(\mu, z_{0}\right)=\frac{W\left(z_{0}\right)}{2 \pi \omega_{K}\left(z_{0}\right)^{2}}
$$

where $\omega_{K}$ is the density of the equilibrium measure with respect to arc length on $\partial K$ (note that the equilibrium measure is supported on $\partial K$ ).

\section{Lubinsky's universality on general sets}

Let $\mu$ be a measure with compact support on the real line, and for simplicity let us assume that $d \mu(x)=w(x) d x$ with an $L^{1}$ function $w$. A form of universality in random matrix theory/statistical quantum mechanics can be expressed via orthogonal polynomials in the form (recall that $K_{n}$ are the reproducing kernels from (21))

$$
\lim _{n \rightarrow \infty} \frac{K_{n}\left(x+\frac{a}{w(x) K_{n}(x, x)}, x+\frac{b}{w(x) K_{n}(x, x)}\right)}{K_{n}(x, x)}=\frac{\sin \pi(a-b)}{\pi(a-b)} .
$$

(The term "universality" comes from the fact that the right-hand side is independent of the original weight $w$ as well as of the place $x$ ). There has been a lot of papers devoted to universality both in the mathematics and in the physics literature; the very frst instance is due to E. Wigner concerning the Hermite weight. Previous approaches used rather restrictive assumptions, see [16] for references. In [16] D. S. Lubinsky recently gave a stunningly simple approach that proves (28) for measures in the $\operatorname{Reg}$ class for which $\operatorname{supp}(\mu)=[-1,1]$ and $w$ is continuous and positive on an interval $I$ (then (28) holds on $I$ uniformly in $|a|,|b| \leq A$, for any $A>0$ ). In [41], again with the polynomial inverse image method, universality was extended to regular measures with arbitrary support (the same result was proved by B. Simon in [32] using so called Jost solutions to recurrences):

Theorem 9.1 (28) holds uniformly in $|a|,|b| \leq A, A>0$ at every continuity point of the weight $w$ (lying inside the support) provided $d \mu(x)=w(x) d x$ is in the Reg class.

When the support is $[-1,1]$, the almost every version of (28) under the local Szeg $\varnothing$ condition $\log w \in L^{1}(I)$ was proved in [10], which just pulls over to the general case (the support arbitrary) via the polynomial inverse image method (see [41]).

Theorem 9.2 (28) holds at almost every point of an interval I provided $d \mu(x)=$ $w(x) d x$ is in the $\mathbf{R e g}$ class and $\log w \in L^{1}(I)$. 


\section{Fine zero spacing of orthogonal polynomials}

Let $\mu$ be a measure with compact support on the real line, and let $p_{n}=p_{n}(\mu, z)$ be the $n$-th orthonormal polynomial with respect to $\mu$. It is well known that classical orthogonal polynomials on $[-1,1]$ have rather uniform zero spacing: if $x_{n, j}=\cos \theta_{n, j}$ are the zeros of the $n$-th orthogonal polynomials, then (inside $(-1,1)$ ) $\theta_{n, j}-\theta_{n, j+1} \sim 1 / n$. In turn, this property of zeros is of fundamental importance in quadrature and Lagrange interpolation. Several hundreds of papers have been devoted to zeros of orthogonal polynomials, still the following beautiful result has only been proven a few years ago, namely when Levin and Lubinsky [15] found that Lubinsky's universality described in Section 9 implies very £ne zero spacing:

$$
\lim _{n \rightarrow \infty}\left(x_{n, k+1}-x_{n, k}\right) \frac{n}{\pi \sqrt{1-x_{n, k}^{2}}}=1 .
$$

With the polynomial inverse image method this was extended in [41] to arbitrary support (see also [32]):

Theorem 10.1 If $K=\operatorname{supp}(\mu) \subset \mathbf{R}, \mu \in \mathbf{R e g}$ and $\mu^{\prime}$ is continuous and positive about $x$, then

$$
\lim _{n \rightarrow \infty} n\left(x_{n, k+1}-x_{n, k}\right) \omega_{K}(x)=1, \quad\left|x_{n, k}-x\right| \leq A / n
$$

where $\omega_{K}$ is the density of the equilibrium measure of the support $K$.

Furthermore, this holds locally a.e. under the local Szegb condition $\log \mu^{\prime} \in L^{1}$ :

Theorem 10.2 If $K=\operatorname{supp}(\mu) \subset \mathbf{R}, \mu \in \mathbf{R e g}$ and $\log \mu^{\prime} \in L^{1}(I)$ for some interval $I$, then (30) is true a.e. in I in the sense that for almost every $x \in I$ and for every $A>0$ we have (30) for $\left|x_{n, k}-x\right| \leq A / n$.

\section{Polynomial approximation on compact subsets of the real line}

The approximation of the $|x|$ function on $[-1,1]$ by polynomials is a key to many problems in approximation theory. Let $E_{n}(f, F)$ denote the error of best approximation to $f$ on $F$ by polynomials of degree at most $n$. S. N. Bernstein [3] proved in 1914 , that the limit

$$
\lim _{n \rightarrow \infty} n E_{n}(|x|,[-1,1])=\sigma
$$

exists, it is £nite and positive. This is a rather diffcult result (with a proof over 50 pages). For $\sigma$ he showed $0.278<\sigma<0.286$. The exact value of $\sigma$ is still unknown. Bernstein returned to the same problem some 35 years later in [4], [5], and he established that for $p>0, p$ not an even integer, the $£$ nite and nonzero limit

$$
\lim _{n \rightarrow \infty} n^{p} E_{n}\left(|x|^{p},[-1,1]\right)=\sigma_{p}
$$


exists, furthermore that for $x_{0} \in(-1,1)$

$$
\lim _{n \rightarrow \infty} n^{p} E_{n}\left(\left|x-x_{0}\right|^{p},[-1,1]\right)=\left(1-x_{0}^{2}\right)^{p / 2} \sigma_{p}
$$

holds true, where $\sigma_{p}$ is the same constant as in (32).

In this section we discuss the problem that arises for more general sets. This problem was considered by R. K. Vasiliev in [44]. His approach is as follows. Let

$$
F=[-1,1] \backslash \cup_{i=1}^{\infty}\left(\alpha_{i}, \beta_{i}\right)
$$

and form the sets

$$
F_{m}=[-1,1] \backslash \cup_{i=1}^{m-1}\left(\alpha_{i}, \beta_{i}\right) .
$$

$F_{m}$ consists of $m$ intervals

$$
F_{m}=\cup_{j=1}^{m}\left[a_{j}, b_{j}\right]
$$

$a_{1}<b_{1}<a_{2}<b_{2} \cdots b_{m-1}<a_{m}<b_{m}$, and for it de£ne

$$
h_{F_{m}}(x)=\frac{\prod_{j=1}^{m-1}\left|x-\lambda_{j}\right|}{\sqrt{\prod_{j=1}^{m}\left|x-a_{j}\right|\left|x-b_{j}\right|}},
$$

where $\lambda_{j}$ are chosen so that

$$
\int_{b_{k}}^{a_{k+1}} \frac{\prod_{j=1}^{m-1}\left(t-\lambda_{j}\right)}{\sqrt{\prod_{j=1}^{m}\left|t-a_{j}\right|\left|t-b_{j}\right|}} d t=0
$$

for all $k=1, \ldots, m-1$. Set

$$
h_{F}(x)=\lim _{m \rightarrow \infty} h_{F_{m}}(x)=\sup _{m} h_{F_{m}}(x),
$$

where it can be shown that the limit exists (but it is not necessarily fnite).

Now with these notations Vasiliev claims the following two results:

$$
\begin{gathered}
\lim _{n \rightarrow \infty} n^{p} E_{n}\left(\left|x-x_{0}\right|^{p}, F\right)=h_{F}\left(x_{0}\right)^{-p} \sigma_{p}, \\
\lim _{n \rightarrow \infty} n^{p} E_{n}\left(\left|x-x_{0}\right|^{p}, F\right)>0 \Longleftrightarrow \int_{0}^{1} \frac{\operatorname{meas}\left\{\left[x_{0}-t, x_{0}+t\right] \backslash F\right\}^{2}}{t^{3}} d t<\infty .
\end{gathered}
$$

This second claim seems to contradict the fact (see e.g. [40, Corollary 10.4]) that there are (Cantor type) sets of measure zero for which $E_{n}\left(\left|x-x_{0}\right|^{p}, F\right) \geq c n^{-p}$ with some $c>0$ (for a set $F$ of zero measure the integral is clearly infnite). Vasiliev's paper [44] is 166 pages long, and it is dedicated solely to the proof of (34) and (35), so it is diffcult to say what might be wrong in the proof. We do not know if the full (34) is correct, but we gave in [40, Theorem 10.5] a few pages proof, based on polynomial inverse images, that shows its validity provided $x_{0}$ lies in the interior of 
$E$. In fact, in this case we have transferred the original Bernstein theorem (32) into Vasiliev's theorem.

Taking into account the form (40) of the equilibrium measure for several intervals, we see that Vasiliev's function is just $h_{F}(x)=\pi \omega_{F}(x)$ if $F$ consists of a £nite number of intervals (and also if $F$ is arbitrary compact, but $x$ is in its interior). Hence, (34) for $x_{0} \in \operatorname{Int}(F)$ takes the following form.

Theorem 11.1 (R. K. Vasiliev) Let $F \subseteq \mathbf{R}$ be compact and let $x_{0}$ be a point in the interior of $F$. Then

$$
\lim _{n \rightarrow \infty} n^{p} E_{n}\left(\left|x-x_{0}\right|^{p}, F\right)=\left(\pi \omega_{F}\left(x_{0}\right)\right)^{-p} \sigma_{p}
$$

where $\sigma_{p}$ is the constant from Bernstein's theorem (32).

E.g. if $F=[-1,1]$, then

$$
\pi \omega_{[-1,1]}(x)=\frac{1}{\sqrt{1-x^{2}}},
$$

and in this special case we recapture Bernstein's result (33).

Here again, Theorem 11.1 can be obtained from Bernstein's theorems (32) via polynomial mappings and approximation.

\section{Appendix: basic notions from logarithmic potential theory}

For a general reference to logarithmic potential theory see [30].

Let $E \subset \mathbf{C}$ be compact. Except for pathological cases, there is a unique probability (Borel) measure $\mu_{E}$ on $E$, called the equilibrium measure of $E$, that minimizes the energy integral

$$
\iint \log \frac{1}{|z-t|} d \mu(z) d \mu(t) .
$$

$\mu_{E}$ certainly exists if $E$ has non-empty interior. One should think of $\mu_{E}$ as the distribution of a unit charge placed on the conductor $E$ (in this case Coulomb's law takes the form that the repelling force between charged particles is proportional with the reciprocal of the distance).

The logarithmic capacity of $E$ is $\operatorname{cap}(E)=\exp (-V)$, where $V$ is the minimum of the energies (37) above. The Green's function of the unbounded component $\Omega$ of the complement $\mathbf{C} \backslash E$ with pole at infnity is denoted by $g_{\Omega}(z, \infty)$, and it has the form

$$
g_{\Omega}(z, \infty)=\int \log \frac{1}{|z-t|} d \mu_{E}(t)+\log \operatorname{cap}(E) .
$$

When $E \subset \mathbf{R}$ then we shall denote by $\omega_{E}(t)$ the density of $\mu_{E}$ with respect to Lebesgue measure wherever it exists. It certainly exists in the interior of $E$. For example 


$$
\omega_{[-1,1]}(t)=\frac{1}{\pi \sqrt{1-t^{2}}}, \quad t \in[-1,1]
$$

is just the well known Chebyshev distribution.

If $E=T_{N}^{-1}[-1,1], E=\cup_{i=1}^{N} I_{i}$ in such a way that $T_{N}$ maps each of the intervals $I_{i}$ onto $[-1,1]$ in a 1 -to-1 way, then (see [13], [30])

$$
\mu_{E}(A)=\frac{1}{N} \sum_{i=1}^{N} \mu_{[-1,1]}\left(T_{N}\left(A \cap I_{i}\right)\right)
$$

which gives

$$
\omega_{E}(t)=\frac{\left|T_{N}^{\prime}(t)\right|}{\pi N \sqrt{1-T_{N}(t)^{2}}}, \quad t \in E .
$$

We also know a rather explicit form for $\omega_{E}$ when $E=\cup_{1}^{l}\left[a_{j}, b_{j}\right]$ is a set consisting of £nitely many intervals (see e.g. [39]):

$$
\omega_{E}(x)=\frac{\prod_{j=1}^{l-1}\left|x-\lambda_{j}\right|}{\pi \sqrt{\prod_{j=1}^{l}\left|x-a_{j}\right|\left|x-b_{j}\right|}},
$$

where $\lambda_{j}$ are chosen so that

$$
\int_{b_{k}}^{a_{k+1}} \frac{\prod_{j=1}^{l-1}\left(t-\lambda_{j}\right)}{\sqrt{\prod_{j=1}^{l}\left|t-a_{j}\right|\left|t-b_{j}\right|}} d t=0
$$

for all $k=1, \ldots, l-1$. It can be easily shown that these $\lambda_{j}$ 's are uniquely determined and there is one $\lambda_{j}$ on any contiguous interval $\left(b_{k}, a_{k+1}\right)$.

Acknowledgements Supported by NSF DMS0968530

\section{References}

1. M. Baran, Complex equilibrium measure and Bernstein type theorems for compact sets in $R^{n}$, Proc. Amer. Math. Soc., 123(1995), 485-494.

2. D. Benkb and V. Totik, Sets with interior extremal points for the Markoff inequality, J. Approx. Theory 110 (2001), 261-265.

3. S. N. Bernstein, Sur la meilleure approximation de $|x|$ par des polynomes des degrés donnés, Acta Math. (Scandinavian) 37 (1914), 1- 57.

4. S. N. Bernstein, On the best approximation of $|x|^{p}$ by means of polynomials of extremely high degree, Izv. Akad. Nauk SSSR, Ser. Mat. 2 (1938), 160-180. Reprinted in S. N. Bernstein "Collected Works," Vol. 2, pp. 262-272. Izdat. Nauk SSSR, Moscow, 1954. [In Russian]

5. S. N. Bernstein, On the best approximation of $|x-c|^{p}$, Dokl. Akad. Nauk SSSR 18 (1938), 379- 384. Reprinted in S. N. Bernstein "Collected Works," Vol. 2, pp. 273-260. Izdat. Nauk SSSR, Moscow, 1954. [In Russian] 
6. S. N. Bernstein, Extremal properties of polynomials and best approximation of functions of a real variable, I., ONTI, 1-203. [In Russian]

7. A. B. Bogatyrev, Effective computation of Chebyshev polynomials for several intervals, Math. USSR Sb., 190 (1999), 1571-1605.

8. P. Borwein, Markoff's and Bernstein inequalities on disjoint intervals, Canad. J. Math. 33 (1981), 201-209.

9. T. Carleman, Über die Approximation analytischer Funktionen durch lineare Aggregate von vorgegebenen Potenzen, Ark. Mat. Astr. Fys., 17(1923), 215-244.

10. M. Findley, Universality for locally Szegb measures, J. Approx. Theory., 155, 136-154.

11. M. Findley, Fine asymptotics for Christoffel functions for general measures, Trans. Amer. Math. Soc. (to appear)

12. G. Freud, Orthogonal Polynomials, Pergamon Press, Oxford, 1971.

13. J. S. Geronimo and W. Van Assche, Orthogonal polynomials on several intervals via a polynomial mapping, Trans. Amer. Math. Soc. 308 (1988), 559- -581.

14. U. Grenander and G. Szegø, Toeplitz Forms and Their Applications, University of California Press, Berkeley and Los Angeles, 1958.

15. A. L. Levin, and D. S. Lubinsky, Applications of universality limits to zeros and reproducing kernels of orthogonal polynomials, J. Approx. Theory. (to appear)

16. D. S. Lubinsky, A new approach to universality limits involving orthogonal polynomials, $A n$ nals of Math. (to appear)

17. D. S. Lubinsky, Universality limits in the bulk for arbitrary measures on compact sets, J. Anal. Math., 106(2008), 373-394.

18. A. Máté, P. Nevai and V. Totik, Szegø's extremum problem on the unit circle, Annals of Math., 134(1991), 433-453.

19. H. P. McKean and P. van Mooerbeke, Hill and Toda curves, Comm. Pure Appl. Math., 33(1980), 23-42.

20. B. Nagy and V. Totik, Sharpening of Hilbert's lemniscate theorem, J. D'Analyse Math., 96(2005), 191-223.

21. P. Nevai, Géza Freud, orthogonal polynomials and Christoffel functions. A case study, J. Approx. Theory, 48(1986), 1-167.

22. L. A. Pastur, Spectral and probabilistic aspects of matrix models. Algebraic and geometric methods in mathematical physics (Kaciveli, 1993), 207-242, Math. Phys. Stud., 19, Kluwer Acad. Publ., Dordrecht, 1996.

23. F. Peherstorfer, Deformation of minimizing polynomials and approximation of several intervals by an inverse polynomial mapping, J. Approx. Theory 111 (2001), 180-195.

24. F. Peherstorfer, On Bernstein-Szegø orthogonal polynomials on several intervals, I. SIAM J. Math. Anal. 21 (1990), 461-482.

25. F. Peherstorfer, On Bernstein-Szegø orthogonal polynomials on several intervals, II. J. Approx. Theory 64 (1991), 123-161.

26. F. Peherstorfer, Orthogonal and extremal polynomials on several intervals, J. Comp. Applied Math. 48 (1993), 187-205.

27. F. Peherstorfer, Elliptic orthogonal and extremal polynomials, J. London Math. Soc. 70 (1995), 605- 624 .

28. F. Peherstorfer and K. Schiefermayr, Theoretical and numerical description of extremal polynomials on several intervals I, Acta Math. Hungar 83 (1999), 27-58.

29. F. Peherstorfer and R. Steinbauer, On polynomials orthogonal on several intervals, Ann. Num. Math. 2 (1995), 353-370.

30. T. Ransford, Potential Theory in the Complex Plane, Cambridge University Press, Cambridge, 1995.

31. R. M. Robinson, Conjugate algebraic integers in real point sets, Math. Z., 84(1964), 415-427.

32. B. Simon, Two extensions of Lubinsky's universality theorem, J. D'Analyse Math., 105(2008), 345-362.

33. B. Simon, The Christoffel-Darboux kernel, "Perspectives in PDE, Harmonic Analysis and Applications" in honor of V.G. Maz'ya's 70th birthday, to be published in Proceedings of Symposia in Pure Mathematics, 79(2008), 295-335. 
34. B. Simon, Weak convergence of CD kernels and applications, Duke Math. J. 146(2009), 305330.

35. H. Stahl and V. Totik, General Orthogonal Polynomials, Encyclopedia of Mathematics and its Applications, 43, Cambridge University Press, Cambridge, 1992.

36. G. Szegø, Über einen Satz des Herrn Serge Bernstein, Schriften Königsberger Gelehrten Ges. Naturwiss. Kl., 5 (1928/29), 59-70.

37. G. Szegф, Collected Papers, ed. R. Askey, Birkhaüser, Boston-Basel-Stuttgart, 1982.

38. V. Totik, Asymptotics for Christoffel functions for general measures on the real line, $J$. D'Analyise Math., 81 (2000), 283-303.

39. V. Totik, Polynomial inverse images and polynomial inequalities, Acta Math., 187(2001), 139160.

40. V. Totik, Metric properties of harmonic measures, Memoirs of the American Mathematical Society, 184, number 867, 2006

41. V. Totik, Universality and £ne zero spacing on general sets, Arkiv för Math., 47(2009), 361391.

42. V. Totik, Chebyshev constants and the inheritance problem, J. Approx. Theory, 160(2009), $187-201$.

43. V. Totik, Christoffel functions on curves and domains, Trans. Amer. Math. Soc., 362(2010), 2053-2087.

44. R. K. Vasiliev, "Chebyshev Polynomials and Approximation Theory on Compact Subsets of the Real Axis," Saratov University Publishing House, 1998. 